popularity. The combined transarterial and transvenous 'trapping' of the fistula, is challenging and few reports as to seafty and curative are available. We have used the transumbilical arterial approach in newborns with good results. The concept of devascularization in a staged fashion over a period of time, has permit us to get a total or near total obliteration in $80 \%$ of cases. There is however a group of patients in whom the residual arterial supplies is through small perforators and in whom transvenous embolization (TVE) is technichally the easiest for cure. We assessed outcomes of our vein of Galen malformation cases treated with TVE.

Materials and Methods We retrospectively reviewed 141 consecutive patients with VGAM treated in our institute between 2004 and 2019. Thalamic AVMs were excluded. 9 patient underwent TVE.

Results Among 9 TVE, 1 underwent partial TVE for palliative measures and excluded from this analysis. The other 8 underwent TVE. All of them had undergone more than 3 transarterial nBCA embolizations. 4 out of 8 patients had complete obliteration of the VOGM. 2 patients underwent stereotactic radiosurgery for the residual fistula and complete obliteration was confirmed (prior to 2000). The outcome 6 out of 8 patient head total obliteration, and achieved normal development without neurological deficit. On the other hand, two hemorrhagic complications occured, 1 imediatelly after the procedure (2013), and the 2nd within 2 days after TVE (2018), where there was a small residual. Of them, the 1st expired and the 2 nd had significant neurological impairment. Both had coils embolization. 2 patients with coils embolization were cured. This difficulty in predicting the risk of hemorrhage, prompted us to improve our ability to close the shunt, and the small remaining arterial supply by using the Chapot 'Pressure cooker' (CHPC) technique in 2 with compleat obliteration.

Discussion There has not been any report before, about the TVE to cure the VGAM after multiple sessions of TAE. In this study, two issues can be brought up. One, there is a chance to close the fistula compleatelly or incompletely only using coils. In addition there is the concern of imparing drenage of the normal brain. To improve these two issues, complete closure of the fistula using the CHPC technique with liquid embolic material in TVE, which we performed recently with great success, seems to be a solution.

Conclusion In endovascular treatment of the vein of Galen malformation, TVE is feasible option, once the dilated vein of Galen becomes small enough. To prevent incomplete occlusion or post procedural hemorrhagic complication, the use of the Chapot 'Pressure cooker' technique using liquid embolic material is considered to be necessary.

Disclosures T. Shigematsu: None. A. Berenstein: None.

\section{E-150 SAFETY AND EFFICACY OF HIGH DOSE INTRAARTERIAL VASODILATORS FOR VASOSPASM AND THE PREVENTION OF DELAYED CEREBRAL ISCHEMIA: COMPARISON OF NICARDIPINE AND VERAPAMIL}

${ }^{1} \mathrm{~T}$ White ${ }^{*},{ }^{1} \mathrm{~J}$ Turpin, ${ }^{1} \mathrm{~K}$ Shah, ${ }^{1} \mathrm{~A}$ Dehdashti, ${ }^{2} \mathrm{~J}$ Katz, ${ }^{1} \mathrm{H}$ Woo. ${ }^{1}$ Neurosurgery, Northwell Health, Manhasset, NY; ${ }^{2}$ Neurology, Northwell Health, Manhasset, NY

10.1136/neurintsurg-2020-SNIS.182
Introduction Delayed cerebral ischemia (DCI) continues to be a challenging complication of subarachnoid hemorrhage. This study sought to examine the impact of intra-arterial therapy on patients with medically refractory DCI. More specifically it sought to determine comparative results of nicardipine and verapamil as well as to determine if there was a relationship between IA vasodilator dose and complication rate.

Methods A retrospective chart review of all patients at a single institution undergoing endovascular IA therapy for vasospasm secondary to subarachnoid hemorrhage over a 30 month period was done. In total, 69 patients underwent 126 treatments for cerebral vasospasm in the setting of atraumatic subarachnoid hemorrhage (SAH). In total $91 \%$ of patients present with aneurysmal SAH while $9 \%$ had angiography negative SAH. $53.6 \%$ of patients had their aneurysm treated endovascularly. Median Hunt Hess grade was 3 and $88 \%$ of patients presented with Modified Fischer grade 3 or 4 . Formal angiography was done in the setting of clinical vasospasm as determine by patients' neurologic exams, velocity on transcranial doppler, and other clinical factors. The majority of patients underwent a single treatment $(55 \%)$ while the most treatments for a single patient was $5(4 \%)$.

Results In total, 98\% (123/126) of treatments led to an improved or stable neurological exam post treatment, and 3 patients developed a post-operative procedural neurological decline. Delayed cerebral ischemia, defined as a new neurological deficit, occurred in 55\% (38/69) of patients in this cohort; however, the new neurological deficit was permanent in only $14 \%$ of patients (10/69). New infarcts were seen on imaging in $19 \%$ of patients $(13 / 69)$ but were clinically silent in $3 / 60$ patients. Patients with DCI were more likely to undergo multiple treatments of IA therapy $(\mathrm{P}<0.05)$. Average total dose of nicardipine was $11 \mathrm{mg}$ (range 3-40 mg). Average dose of verapamil was $88 \mathrm{mg}$ (range $25-240 \mathrm{mg}$ ). There was no relationship between dose and complication rate $(\mathrm{P}>0.05)$. There was no differential risk of DCI in the nicardipine vs the verapamil group $(\mathrm{P}>0.05)$. Patients undergoing verapamil therapy were more likely to experience complications of IA therapy, $20 \%$ vs $5 \%$ in the nicardipine group $(\mathrm{P}<0.05)$, however patients classified to have severe vasospasm by the angiographer were more likely to undergo treatment with verapamil $(\mathrm{P}<0.05)$.

Conclusion This report adds to the growing body of literature that endovascular rescue therapy offers a safe and effective option in managing medically refractory vasospasm in order to prevent DCI. Importantly, it also suggests that in select patients high dose therapy can be used for maximal benefit with a reasonable side effect profile. Importantly, this series also demonstrates an initial rate of DCI of $55 \%$ in patients with vasospasm undergoing IA therapy with only $14 \%$ of patient's demonstrating permanent neurological deficit suggesting a significant overall benefit of treatment. The results of this initial study mandate further investigation into the safety and efficacy of intra-arterial verapamil and nicardipine for the treatment and prevention of DCI. More importantly it shows that dose optimization needs to be done prior to pursuing further trials.

Disclosures T. White: None. J. Turpin: None. K. Shah: None. A. Dehdashti: None. J. Katz: None. H. Woo: None. 